\title{
LDPC-based Joint Source-Channel-Network Coding for the Multiple Access Relay Channel
}

\author{
Marwa Ben Abdessalem*, Amin Zribi*†, Tadashi Matsumoto ${ }^{\ddagger}$, and Ammar Bouallègue* \\ ${ }^{*}$ University of Tunis El Manar, National Engineering School of Tunis, SysCom laboratory, BP 37, Le Belvedere, 1002, Tunis \\ Email: marwa.benabdessalem@enit.utm.tn, ammarbouallegue.syscom@gmail.com \\ $\dagger$ IMT Atlantique, Lab-STICC, UBL, 29238 Brest, France \\ Email: amin.zribi@isetcom.tn \\ $\ddagger$ Japan Advanced Institute of Science and Technology,1-1 Asahidai,Nomi,Ishikawa, Japan, \\ and Centre for Wireless Communications (CWC), University of Oulu, Finland (Part-time) \\ Email:matumoto@jaist.ac.jp
}

\begin{abstract}
In this work, we investigate the MARC (Multiple Access Relay Channel) setup, in which two Markov sources communicate to a single destination, aided by one relay, based on Joint Source Channel Network (JSCN) LDPC codes. In addition, the two source nodes compress the information sequences with an LDPC source code. The compressed symbols are directly transmitted to both a relay and a destination nodes in two transportation phases. Indeed, the relay performs the concatenation of the received compressed sequences to obtain a recovered sequence, which is encoded with an LDPC channel code, before being forwarded to the destination. At the receiver, we propose an iterative joint decoding algorithm that exploits the correlation between the two sources-relay data and takes into account the errors occurring in the sources-relay links to estimate the source data. We show based on simulation results that the JSCN coding and decoding scheme into a MARC setup achieves a good performance with a gain of about $5 \mathrm{~dB}$ compared to a conventional LDPC code.
\end{abstract}

Keywords: Joint Source Channel Network scheme, LDPC codes, MARC system.

\section{INTRODUCTION}

In the framework of wireless sensor networks, cooperation nodes aim to improve the network performance by providing diversity gains through cooperation. Recently, there has been a great interest for the cooperative communication structures. One of these structures is the Multiple Access Relay Channel (MARC). The MARC consists of two sources (e.g. users) or more that send data with the help of a single relay to communicate with a single destination. In [1], authors expressed the outer bounds of capacity for the MARC and since, many practical coding solutions were investigated.

In the literature, several relaying operations, for instance AF (Amplify and Forward), CF (Compress and Forward), and DF (Decode and Forward) are proposed and theoretically investigated for the application to the MARC respectively in [2]-[4]. Also, the different relaying operations were considered in many practical coding approaches design [5], [6]. Motivated by the $\mathrm{CoF}$ (compute and forward) operation mode, authors in [7] proposed a new coding design for the MARC. Also, in [8], the QF (quantize and Forward) relaying operation was proposed with multiple number of transmitters.

Nowadays, Low-Density-Parity-Check (LDPC) codes have attracted much interest for several wireless communication systems, due to their simple construction and their efficient decoding process that results in low error rates over Additive White Gaussian Noise (AWGN) channels that is close to the Shannon limit [9]. Hence, several contributions considered LDPC codes for the MARC setup. In [10], L. Liu et al considered a new distributed superposition coding method for an asymmetric SW (Slepian-Wolf) MARC system based on Joint Source Channel (JSC) LDPC codes. Also, LDPC coding scheme was investigated in [11] for the MARC, where the information sequences from the two sources are re-encoded by the relay node separately which then effectuate a network coding. Furthermore, several channel coding network schemes are developed over MARC with other channel codes such as accumulator (ACC) [12], turbo codes [13], and product codes [14]. In [15], authors proposed the transmission of two correlated binary and independent sources using the lossy forward (LF) relaying strategy using ACC. In [16], authors proposed a MARC setup to transmit a correlated content using serially concatenated codes. One common feature of all these contributions mentioned above is that no source compression scheme is applied. Indeed, according to our knowledge few contributions were investigating the source compression coding in the framework of the network coding [17]. In general, variable length entropy coding techniques for the source coding are not suitable since they render the compressed bitstream transfer very sensitive to channel noise. Therefore, a JSC coding scheme was proposed in [18] based on double LDPC code, where the source code with a fixed length use an LDPC code to compress a redundant information sequence, concatenated with an LDPC code to protect against channel impairments the corresponding compressed information. The double LDPC system is modeled by a single Tanner graph (concatenation between source and channel graphs), which is then decoded with the Belief Propagation (BP) algorithm [19], 


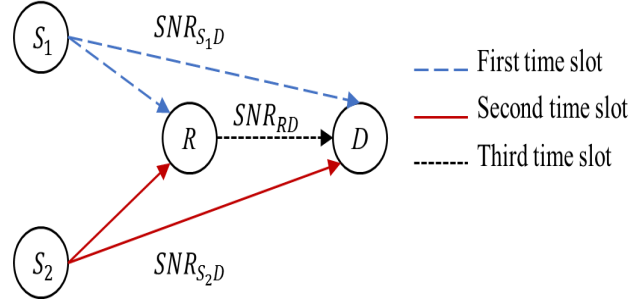

Fig. 1. The MARC model.

[20].

The main contributions behind this work are: first, a coding and decoding solution based on Joint Source Channel Network (JSCN) is proposed into a two sources MARC setup. We introduce the LDPC source code in both the source nodes and the LDPC channel code into the relay node. We assume that the relay observes a corrupted data from the two sources. Second, we propose a joint decoding process, that consists of three Tanner graphs combined with a BCJR algorithm to exploit the sources/relay correlations and the residual redundancy to enhance the MARC system performance.

As following of this paper, we present in Section II the system model and notations. We describe in Section III the proposed joint decoder. We outline in Section IV the results of simulation. Lastly, we give in Section V a general conclusion of this work.

\section{SYSTEM MODEL AND NOTATIONS}

We investigate in this paper, the case where two source nodes want to deliver a correlated content to a receiving node through cooperation (relay node). Hence, as shown in Fig. 1 , the MARC is composed of $S_{1}$ and $S_{2}$ sources, a single relay $R$, and a single destination $D$. Moreover, we assume that no communication between the two sources is associated. The JSCN LDPC codes are considered for both compression, error protection and network coding. In the proposed system, these functions are distributed between the $S_{1}$ and the $S_{2}$ nodes, and the $R$ node, respectively. In Fig. 2, we show the MARC system block diagram for the proposed JSCN. The data sequences $\mathbf{u}_{1}$ and $\mathbf{u}_{2}$ generated from $S_{1}$ and $S_{2}$, respectively are independent but internally correlated. $\mathbf{u}_{1}$ with $n_{1}$ symbols length, $\mathbf{u}_{2}$ is then with $n_{2}$ symbols length. Each correlated source is modelled as a Markov source $\left(S_{t}\right)$, described by $\alpha=\operatorname{Pr}\left(S_{t}=1 \mid S_{t-1}=0\right)$ and $\beta=\operatorname{Pr}\left(S_{t}=0 \mid S_{t-1}=1\right)$, such as $\alpha$ and $\beta$ are the corresponding transition probabilities. We define by $H(S)=\mu_{0} h(\alpha)+\mu_{1} h(\beta)$, such as $h(x)=$ $-x \log _{2}(x)-(1-x) \log _{2}(1-x)$ the Markov source entropy rate, where the stationary distribution are given by $\mu_{0}=1$ $\mu_{1}=\frac{\beta}{\beta+\alpha}$. Also, we consider that the distance between $S_{1}$ and $D$ is equal to the distance between $S_{2}$ and $D,\left(d_{S_{1} D}=\right.$ $d_{S_{2} D}=d_{S D}$ ), thus equivalent SNRs, $S N R_{S_{1} D}=S N R_{S_{2} D}=$ $S N R_{S D}$. We define $d_{R D}$ the distance between the nodes $R$

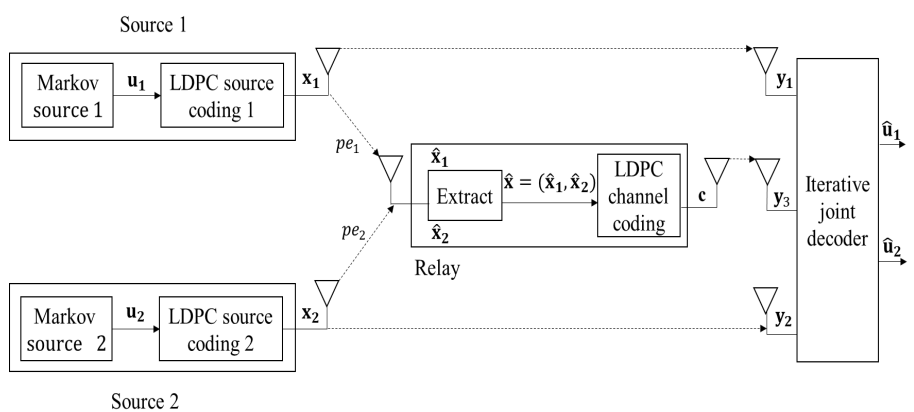

Fig. 2. The MARC system block diagram.

and $D$. Furthermore, $G_{R D}$ is the link gain between $R$ and $D$, defined by $G_{R D}=\left(\frac{d_{S D}}{d_{R D}}\right)^{n}$, where the path-loss exponent $n=3.52$ [21]. We suppose in this work that the position of the relay is situated closer to the two sources than the destination $D$ with $d_{R D}=\left(\frac{3 d_{S D}}{4}\right)$ and $G_{R D}=\left(\frac{d}{3 d / 4}\right)^{3.52}$ $=4.4 \mathrm{~dB}$, where $S N R_{R D}=S N R_{S D}+4.4 \mathrm{~dB}$. We have three communication phases divided into three time slots.

In the first time slot, the first Markov source $S_{1}$ generates the sequence $\mathbf{u}_{1}$. Then, this sequence is compressed by an LDPC source code using $\left(l_{1} \times n_{1}\right)$ parity check matrix $\mathbf{H}_{s c_{1}}$ of the source code as

$$
\mathbf{b}_{1}=\mathbf{H}_{s c_{1}} \times \mathbf{u}_{1}
$$

The sequence $\mathbf{b}_{1}$ is then modulated by BPSK with $l_{1}$ symbols length, to have the signal $\mathbf{x}_{1}$. The source $S_{1}$ node sends the obtained signal $\mathbf{x}_{1}$ to the relay and the destination. We define by $R_{s_{1}}=l_{1} / n_{1}$, the compression rate of the source $S_{1}$. In the second time slot, the sequence $\mathbf{u}_{2}$ generated by the second source $S_{2}$, is compressed by a another LDPC source code with matrix $\mathbf{H}_{s c_{2}}$ of $\left(l_{2} \times n_{2}\right)$ dimensions as,

$$
\mathbf{b}_{2}=\mathbf{H}_{s c_{2}} \times \mathbf{u}_{2}
$$

Then, the sequence $\mathbf{b}_{2}$ is modulated to have the signal $\mathbf{x}_{2}$ with $l_{2}$ bits, which is transmitted to both a relay node and a receiver node. The compression rate of the source $S_{2}$ is $R_{s_{2}}=l_{2} / n_{2}$. The relay node performs the concatenation of the recovered sequences $\hat{\mathbf{x}_{1}}$ and $\hat{\mathbf{x}_{2}}$ during the last time slot as $\hat{\mathbf{x}}=\left(\hat{\mathbf{x}_{1}}, \hat{\mathbf{x}_{2}}\right)$. Using the generator matrix $\mathbf{G}_{c c}^{T}$ of the channel LDPC code, the sequence $\hat{\mathbf{x}}$ is encoded according to the following expression

$$
\mathbf{c}=\mathbf{G}_{c c}^{T} \times \hat{\mathbf{x}}
$$

The encoded sequence c with $m$ bits length is transmitted directly to a destination after modulation through the AWGN channel. We note that $R_{c}=\left(l_{1}+l_{2}\right) / m=l_{3} / m$ is the system channel coding rate. Furthermore, for the MARC system, the overall rate is equal to $R=1 /\left(R_{s}+1 / R_{c}\right)$, where $R_{s}=R_{s_{1}}=$ $R_{s_{2}}$.

The signals $\mathbf{y}_{1}, \mathbf{y}_{2}$, and $\mathbf{y}_{3}$ from the first source $S_{1}$, the second source $S_{2}$, and the relay $R$ are received respectively, the destination $D$ executes the joint decoder to get the original data sequences estimates $\hat{\mathbf{u}_{1}}$ and $\hat{\mathbf{u}_{2}}$. The JSCN decoder is 


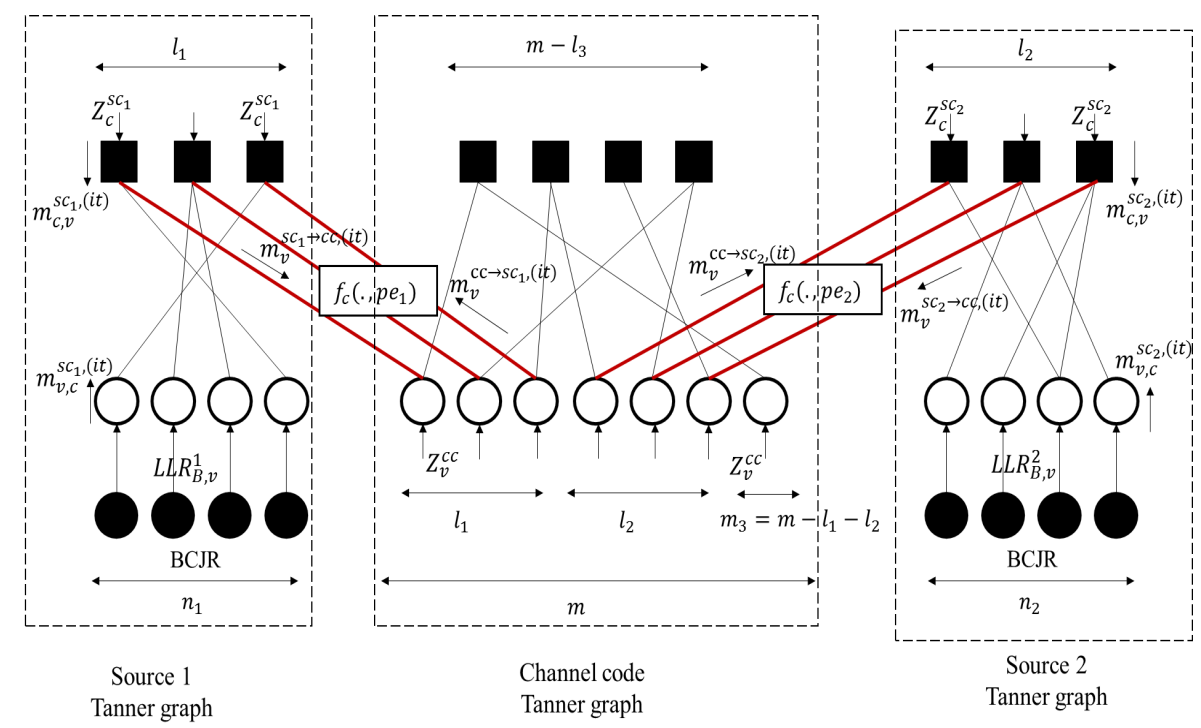

Fig. 3. The proposed JSC decoder for the JSCN coding scheme applied to the MARC network.

represented in Fig. 3 as three bipartite Tanner graphs, and will be detailed in the next section.

\section{THE JSCN ITERATIVE DECODING SCHEME}

The joint decoder based on the message passing BP algorithm for the MARC system is described in this section with LDPC compression and error correction codes. As shown in Fig. 3, the joint decoder based on the LDPC codes presented by three concatenated Tanner graphs. Also, we apply the BCJR algorithm for each LDPC source decoder at the variable nodes to exploit the residual sources correlations. We denote that the iterative decoding involves two types of iteration: JSC LDPC iterations and BCJR source decoding operation. Every $i t_{s c}$ JSC LDPC iterations, the BCJR is activated. The number of BCJR iterations is denoted $i t_{B C J R}$ which resulting in $i t=i t_{B C J R} \times$ $i t_{s c}$ overall iterations. Let us consider the decoder at the $i t$ th iteration. First, in the source $S_{1}$ decoder, the variable nodes $v$ send the corresponding log-likelihood ratios (LLRs) to the check nodes $c$. Therefore, for $v=\left(1, \ldots, n_{1}\right)$, we have

$$
m_{v, c}^{s c_{1},(i t)}=\sum_{c^{\prime} \neq c} m_{c^{\prime}, v}^{s c_{1},(i t-1)}+L L R_{B, v}^{1}
$$

In the same way, the LLRs of the variable nodes $v$ in the $S_{2}$ Tanner graph are sent to the check nodes for $v=\left(1, \ldots, n_{2}\right)$ as

$$
m_{v, c}^{s c_{2},(i t)}=\sum_{c^{\prime} \neq c} m_{c^{\prime}, v}^{s c_{2},(i t-1)}+L L R_{B, v}^{2}
$$

We consider symmetric Markov source, hence, the variable nodes of the source $S_{1}$ decoder and the source $S_{2}$ decoder are initialized by $L L R_{B, v}^{1,2}=0.00$, respectively. After $i t_{s c}$ iterations of the joint decoding process, $L L R_{B, v}^{1,2}$ equal to the extrinsic information delivered by the BCJR algorithm. $\sum_{c^{\prime} \neq c} m_{c^{\prime}, v}^{s c_{1},(i t-1)}$ are the LLRs passed from $c$ (check nodes) to $v$ (variable nodes) in the source $S_{1}$ decoder. While, in the source $S_{2}$ decoder, we consider $\sum_{c^{\prime} \neq c} m_{c^{\prime}, v}^{s c_{2},(i t-1)}$, the LLRs transfer from $c$ to $v$.

In the LDPC channel graph, the first $v$ nodes of length $l_{1}$, calculate their messages as

$$
m_{v, c}^{c c,(i t)}=Z_{v}^{c c}+f_{c}\left(m_{v}^{s c_{1} \rightarrow c c,(i t-1)}, p_{e_{1}}\right)+\sum_{c^{\prime} \neq c} m_{c^{\prime}, v}^{c c,(i t-1)}
$$

where $Z_{v}^{c c}=\frac{2 r_{v}}{\sigma_{R D}^{2}}$ are the LLRs of the channel decoder, which are used to initialize the variable nodes. We note that $\sigma_{R D}^{2}$ is the $R-D$ channel noise variance and the received vector expression is $r_{v}=\left(1-2 c_{v}\right)+n_{v} \cdot m_{v}^{s c_{1} \rightarrow c c,(i t-1)}$ are the messages sent from the source $S_{1}$ decoder by the $c$ nodes to the $v$ nodes on the side of the channel graph.

Also, we assume that the wireless $S_{1}-R$ link is noisy, hence, the sequence $\hat{\mathbf{x}_{1}}$ at the relay node from $S_{1}$ can be damaged by errors with a probability of error $p e_{1}$. In fact, to estimate the sequence $\mathbf{x}_{1}$, the channel graph decoder applies the updating function $f_{c}$ using the LLRs of the sequence $\hat{\mathbf{x}}_{1}$ and the corresponding $p e_{1}$. So, for $i=\left(1, \ldots, l_{1}\right)$, thus for the first $l_{1}$ variable nodes of the LDPC channel code, we apply the following LLRs update:

$$
\begin{aligned}
\operatorname{LLR}\left(x_{1 i}\right) & =\log \left(\frac{\exp \left(\operatorname{LLR}\left(\hat{x}_{1 i}\right)\right)\left(1-p e_{1}\right)+p e_{1}}{\left(1-p e_{1}\right)+\exp \left(\operatorname{LLR}\left(\hat{x}_{1 i}\right)\right) p e_{1}}\right) \\
& =f_{c}\left(\operatorname{LLR}\left(\hat{x}_{1 i}\right), p e_{1}\right)
\end{aligned}
$$

In the same way, we suppose that the $S_{2}-R$ link is noisy for $i=\left(1, \ldots, l_{2}\right)$. Therefore, the sequence $\hat{\mathbf{x}_{2}}$ is corrupted at the relay node with a certain probability of error $p e_{2}$.

Also, the messages of the channel graph for $l_{2}$ variable nodes are

$$
m_{v, c}^{c c,(i t)}=Z_{v}^{c c}+f_{c}\left(m_{v}^{s c_{2} \rightarrow c c,(i t-1)}, p_{e_{2}}\right)+\sum_{c^{\prime} \neq c} m_{c^{\prime}, v}^{c c,(i t-1)}
$$

where $m_{v}^{s c_{2} \rightarrow c c,(i t-1)}$ are the messages passed since the source $S_{2}$ graph by the $c$ nodes to $v$ nodes in the LDPC channel graph. 
Then, in the channel graph, the messages of the remaining $m_{3}$ variable nodes are

$$
m_{v, c}^{c c,(i t)}=Z_{v}^{c c}+\sum_{c^{\prime} \neq c} m_{c^{\prime}, v}^{c c,(i t-1)}
$$

We note that $m_{c^{\prime}, v}^{c c,(0)}=0, m_{c^{\prime}, v}^{s c c_{1},(0)}=0, m_{c^{\prime}, v}^{s c_{2},(0)}=0$, $m_{v}^{s c_{1} \rightarrow c c,(0)}=0$, and $m_{v}^{s c_{2} \rightarrow c c,(0)}=0$.

For each decoder, the LLRs between the $c$ and the $v$ nodes are described as follows. First, for $c=\left(1, \ldots, l_{1}\right)$, the messages of the $S_{1}$ decoder are

$$
\begin{aligned}
m_{c, v}^{s c_{1},(i t)}=2 \tanh ^{-1} & \left(\tanh \left(\frac{Z_{c}^{s c_{1}}}{2}\right) \prod_{v^{\prime} \neq v} \tanh \left(\frac{m_{v^{\prime}, c}^{s c_{1},(i t)}}{2}\right)\right. \\
& \left.\times \tanh \left(\frac{f_{c}\left(m_{v}^{c c \rightarrow s c_{1},(i t)}, p_{e_{1}}\right)}{2}\right)\right)
\end{aligned}
$$

where $Z_{c}^{s c_{1}}=\frac{2 r_{c}}{\sigma_{S_{1} D}^{2}}$ are the LLRs used to initialize the check nodes. Notice that $\sigma_{S_{1} D}^{2}$ is the $S_{1}-D$ channel noise variance, and $r_{c}=\left(1-2 x_{1}\right)+n_{c}$ is the received sample.

$$
m_{v}^{c c \rightarrow s c_{1},(i t)}=Z_{v}^{c c}+\sum_{c^{\prime}} m_{c^{\prime}, v}^{c c,(i t-1)}
$$

are the messages between the channel graph and the source $S_{1}$ graph (from $v$ nodes to $c$ nodes, respectively). While for $c=\left(1, \ldots, l_{2}\right)$, in the source $S_{2}$ decoder, the LLRs send from $c$ to $v$ nodes are

$$
\begin{aligned}
m_{c, v}^{s c_{2},(i t)}=2 \tanh ^{-1} & \left(\tanh \left(\frac{Z_{c}^{s c_{2}}}{2}\right) \prod_{v^{\prime} \neq v} \tanh \left(\frac{m_{v^{\prime}, c}^{s c_{2},(i t)}}{2}\right)\right. \\
& \left.\times \tanh \left(\frac{f_{c}\left(m_{v}^{c c \rightarrow s c_{2},(i t)}, p_{e_{2}}\right)}{2}\right)\right)
\end{aligned}
$$

where $Z_{c}^{s c_{2}}=\frac{2 r_{c}}{\sigma_{S_{2} D}}$ are the LLRs for the check nodes with $r_{c}=\left(1-2 x_{2}\right)+n_{c}$ and $\sigma_{S_{2} D}^{2}$ is the $S_{2}-D$ channel noise variance. And

$$
m_{v}^{c c \rightarrow s c_{2},(i t)}=Z_{v}^{c c}+\sum_{c^{\prime}} m_{c^{\prime}, v}^{c c, i t-1)}
$$

represents the messages passed since the LDPC channel graph toward the source $S_{2}$ decoder (from $v$ to $c$ nodes, respectively). Every $c$ node sends its LLR to $v$ node for $c=\left(1, \ldots, m-l_{3}\right)$ in the channel code Tanner graph, as follows:

$$
m_{c, v}^{c c,(i t)}=2 \tanh ^{-1}\left(\prod_{v^{\prime} \neq v} \tanh \left(\frac{m_{v^{\prime}, c}^{c c,(i t)}}{2}\right)\right)
$$

After $i t$ iterations of the decoding process, the joint decoder estimate $\hat{\mathbf{u}_{1}}$ and $\hat{\mathbf{u}_{2}}$ from the $S_{1}$ and the $S_{2}$ respectively.

\section{Simulation Results}

We analyze in this section, the performance of the proposed LDPC-based JSCN coding and decoding applied to MARC system. First, we compare the performance using different configurations of the JSCN. Second, we treat the case of errors may occur in the $S_{1}-R$ and the $S_{2}-R$ links, and we

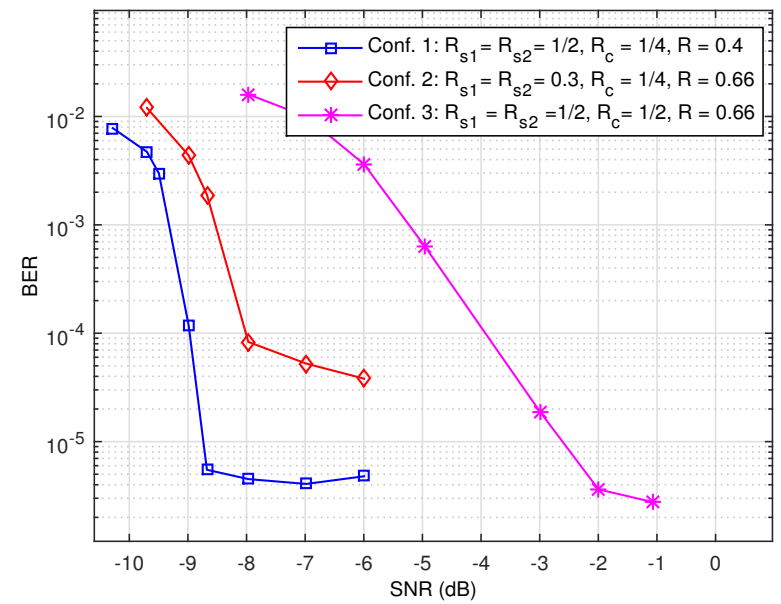

Fig. 4. The performance in terms of BER for the JSCN encoding solution applied to MARC setup.

study the obtained results. For the simulation environment, we consider the simulations Monte-Carlo, using the language C programming.

\section{A. MARC system performance with different source and chan- nel coding rates}

We study the MARC system performance where the wireless links between the two sources-relay are noiseless. The aim of this study is to display the effect of different channel coding and source coding rates on the proposed system using the JSCN LDPC coding. The LDPC codes used in this paper are regular for the sake of simplicity. The design of the LDPC code is not target of this paper and can be considered as the future work. The JSCN decoding process applied the BP algorithm with a global number of iterations $i t=100$, and after $i t_{s c}=20$ iterations, we apply the BCJR algorithm for each source decoder. We note that the complexity of the proposed JSCN decoding scheme is almost the same as the complexity of an LDPC iterative decoder, since similar computational operations are considered for message calculations.

For symmetric Markov source with the transition probabilities $\alpha=\beta=0.07$, we show a BER performance as a function of $S N R_{S D}$ in Fig. 4. We propose to study three rate allocation configurations. The first configuration has rates $R_{c}=1 / 4$ and $R_{s_{1}}=R_{s_{2}}=1 / 2$ for the channel, the source $S_{1}$, and the source $S_{2}$ coding, respectively. The overall transmission rate is equal to $R=0.4$. The second configuration has rates $R_{c}=1 / 4$ and $R_{s_{1}}=R_{s_{2}}=0.3$, hence, an overall rate equal to $R=0.66$. Then, the last one with $R_{c}=1 / 2$, and $R_{s_{1}}=R_{s_{2}}$ $=1 / 2$, which means an overall rate of $R=0.66$. We observe that the first configuration protects more the information with $R_{c}=1 / 4$ than the two others configurations. Therefore, an improvement is shown, for instance $5 \mathrm{~dB}$ succeed compared to the last configuration for a $\mathrm{BER}=10^{-4}$. However, the second scenario allocates less bits and compresses more with source 


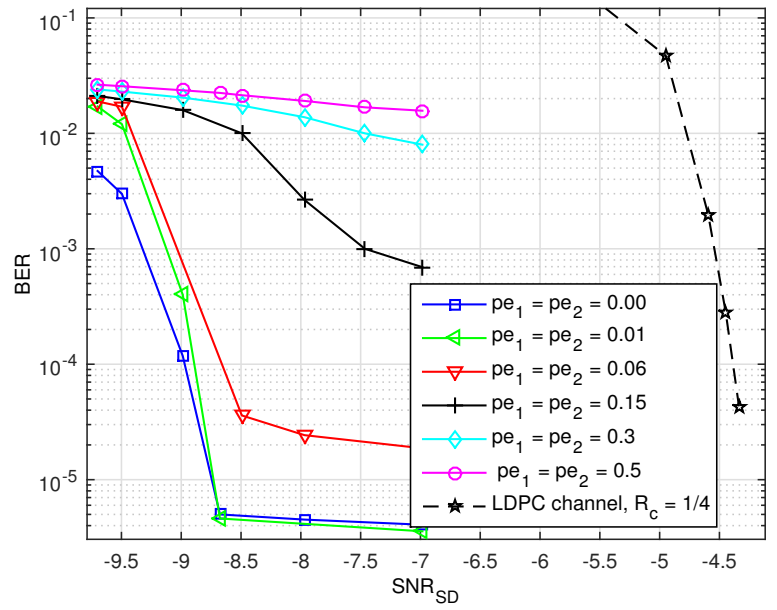

Fig. 5. The performance of JSCN system, where $R_{s_{1}}=R_{s_{2}}=1 / 2, R_{c}=$ $1 / 4$, and different $p e_{1}$ and $p e_{2}$.

coding rates $R_{s_{1}}=R_{s_{2}}=0.3$ than the first one, which induces an error floor increase from almost $5.10^{-6}$ to $5.10^{-5}$. The last code makes less protection than the two other codes with $R_{c}=1 / 2$, hence, in the waterfall region, shows a loss in terms of BER. Using the same source coding rate than the first case, $R_{s_{1}}=R_{s_{2}}=1 / 2$ with the third configuration, we keep almost the same error floor of about $10^{-6}$.

We conclude that the source code rate justifies the system error floor behaviour, and high compression rates makes lower error floor. While, the waterfall system performance depends on the channel code rate and using lower channel coding rates makes more protection, and consequently better performance.

\section{B. JSCN LDPC coding with noisy two sources-relay links}

In this section, we assume that the wireless link $S_{1}-R$ is noisy resulting in an error probability $p e_{1}$, in the same way, we assume that the wireless link $S_{2}-R$ is corrupted with an error probability $p e_{2}$. Also, we note that $S N R_{S_{1} D}=S N R_{S_{2} D}=$ $S N R_{S D}$. The aim behind this simulation is to show the effect of the noisy links error probabilities on the JSCN system.

In Fig. 5, we show a BER performance as a function of $S N R_{S D}$. We can observe that with error probabilities $p e_{1}$ $=p e_{2}=0.01$, we keep almost the same performance in the waterfall region as the case of $p e_{1}=p e_{2}=0.00$. We point out a slight loss of $0.09 \mathrm{~dB}$ for $\mathrm{BER}=10^{-3}$. Also, we can notify that the error floor is the same as the error-free case. we can see that with relatively high error probabilities $p e_{1}=p e_{2}=$ 0.15 , the performance in the waterfall region for a $\mathrm{BER}=$ $10^{-3}$, as well as in the error floor region is destructed, hence, shows a loss of about $1.8 \mathrm{~dB}$. With a high error probabilities of $p e_{1}=p e_{2}=0.5$, and $p e_{1}=p e_{2}=0.3$, the performance is damaged in terms of BER.

Through the direct transmission from the $S_{1}$ and from the $S_{2}$ to the common destination $D$, and the updating function $f_{c}$ applied at the joint decoder to estimate the corrupted sequences at the relay node $R$, the JSCN encoding system persists powerful until a probability of error equal to 0.06 , where just $0.3 \mathrm{~dB}$ are lost compared to the error-free case.

On the other hand, we study a comparison between the JSCN encoding scheme and the standard LDPC channel encoding with channel coding rate $R_{c}=1 / 4$. We observe that the performance of the JSCN system is well than the LDPC code with different error probabilities in the waterfall region with an improvement of about $5 \mathrm{~dB}$.

\section{BER performance of asymmetric error probabilities for sources $S_{1}$ and $S_{2}$}

The idea behind this simulation is to study the performance of the Markov source $S_{1}$ and $S_{2}$ of the proposed system, using asymmetric error probabilities $p e_{1}$ and $p e_{2}$ between $S_{1}-R$ and $S_{2}-R$ links, respectively. For this aim, we keep the same JSCN coding/decoding system presented above where the rates $R_{s_{1}}$ $=R_{s_{2}}=1 / 2$ and $R_{c}=1 / 4$ for the source $S_{1}$, the source $S_{2}$, and the LDPC channel coding, respectively. Also, we consider four cases. The first case with symmetric error probabilities, $p e_{1}=p e_{2}=0.00$. The second case when the $S_{1}-R$ link is corrupted with an error probability $p e_{1}=0.3$, and the $S_{2}-R$ link without error. In the third case, we propose that $p e_{1}=$ 0.06 and $p e_{2}=0.15$, and in the last one, we propose that $p e_{1}$ $=p e_{2}=0.3$.

For the source $S_{1}$, we show a BER performance as a function of $S N R_{S D}$ in Fig. 6. For the source $S_{2}$, we plot the BER with respect to $S N R_{S D}$ in Fig. 7. In Fig. 6, with high error probability $p e_{1}=0.3$, the performance of the source $S_{1}$ is worst in waterfall and error floor regions, since the LLRs of the updating function $f_{c}$ applied at the JSCN decoder are equal to 0.00 , which is not the case for medium-to-low error probabilities. Therefore, comparing with the BER results of the $S_{2}$, the performance is better in the error-free case as shown in Fig. 7. We loose $1 \mathrm{~dB}$ for a $\mathrm{BER}=10^{-3}$ with respect to the performance of the proposed system. In fact, the error floor has increased from almost $5.10^{-6}$ to $2.10^{-5}$. For $p e_{1}=0.06$ and $p e_{2}=0.15$ of the $S_{1}$ and the $S_{2}$, respectively, we can observe almost the same performance with slight improvement in the case of $p e_{1}=0.06$ in Fig. 6. Indeed, having lower error probabilities in the wireless two sources-relay links ( $p e_{1}$ and $p e_{2}$, respectively ) is better in terms of BER performance, than the medium-to-high $p e_{1}$ and $p e_{2}$. Also, the good point is that message passing can help the source having high error probabilities to improve its performance if the second source has a fair channel.

\section{CONCLuSions}

In this contribution, we studied the LDPC-based JSCN scheme applied to MARC setup, which is composed of a single destination, single relay and two Markov sources. First, the corresponding system model is presented, where the source compression and the protection capacity operations are distributed between both sources $S_{1}, S_{2}$ nodes, and the $R$ node respectively. After that, a JSCN decoding scheme is modeled and we presented the message passing algorithm 


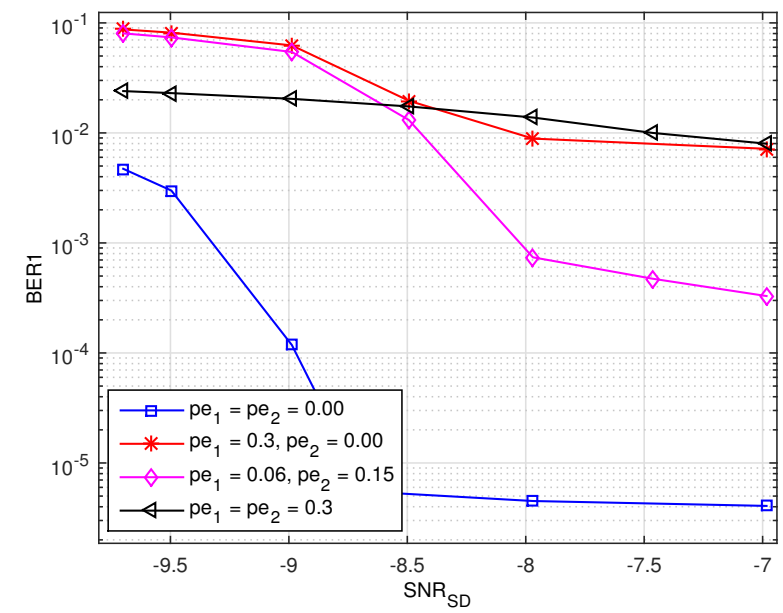

Fig. 6. BER performance for the Markov Source $S_{1}$.

for the proposed JSCN decoder process. Based on simulation results, we showed the performance of the proposed setup with different configurations that depend on source/channel code rates. Then, we studied the performance of the JSCN scheme, assuming errors in sources-relay links with symmetric and asymmetric error probabilities $p e_{1}$ and $p e_{2}$. We distinguish that for medium-to-low $p e_{1}$ and $p e_{2}$ at the $R$ node, the JSCN scheme persists powerful ever for an error probability less than 0.15 .

\section{ACKNOWLEDGEMENT}

This research is in part supported by the Young Researcher Project COMMUN (18PJEC11-01) funded by the Ministry of Higher Education and Scientific Research of Tunisia.

\section{REFERENCES}

[1] L. Sankaranarayanan, G. Kramer, and N. B. Mandayam, "Capacity theorems for the multiple-access relay channel," in Proc. 42nd Annu. Allerton Conf. Communications, Control, and Computing, pp. 1782-1791, 2004.

[2] G. Kramer, M. Gastpar, and P. Gupta, "Cooperative strategies and capacity theorems for relay networks," IEEE Transactions on Information Theory, vol. 51, no. 9, pp. 3037-3063, 2005.

[3] A. Winkelbauer, N. Goertz, and G. Matz, "Compress-and-forward in the multiple-access relay channel: With or without network coding?," 7th International Symposium on Turbo Codes and Iterative Information Processing (ISTC), pp. 131-135, Aug. 2012.

[4] L. Sankar, Y. Liang, N. B. Mandayam, and H. V. Poor, "Fading multiple access relay channels: Achievable rates and opportunistic scheduling," IEEE Transactions on Information Theory, vol. 57, no. 4, pp. 1911-1931, 2011.

[5] M. Hernaez, P. M. Crespo, and J. Del Ser, "On the design of a novel joint network-channel coding scheme for the multiple access relay channel," IEEE Journal on Selected Areas in Communications, vol. 31, no. 8, pp. 1368-1378, 2013.

[6] G. Zeitler, R. Koetter, G. Bauch, and J. Widmer, "On quantizer design for soft values in the multiple-access relay channel," IEEE International Conference on Communications (ICC), 2009.

[7] X. Insausti, A. Sez, and P. M. Crespo, "A novel scheme inspired by the compute-and-forward relaying strategy for the multiple access relay channel," Wireless Networks, pp. 1-9, 2017.

[8] L. Liu, Y. Li, Y. Su, and Y. Sun, "Quantize-and-forward strategy for interleave-division multiple-access relay channel," IEEE Transactions on Vehicular Technology, vol. 65, no. 3, pp. 1808-1814, 2016.

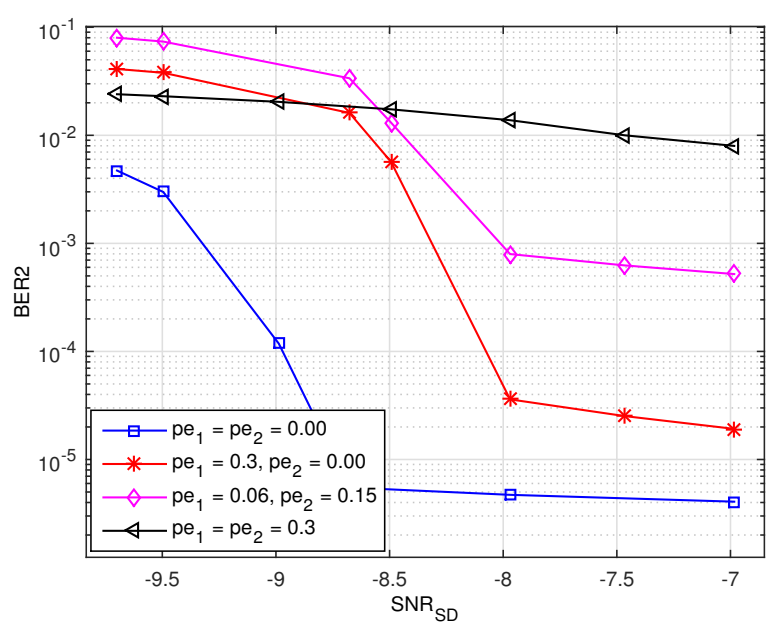

Fig. 7. BER performance of the Markov Source $S_{2}$.

[9] T. J. Richardson, M. A. Shokrollahi, and R. L. Urbanke, "Design of capacity-approaching irregular low-density parity-check codes," IEEE transactions on information theory, vol. 47, no. 2, pp. 619-637, 2001.

[10] L. Liu, Y. Li, C. Yuen, Y. L. Guan, and Y. Sun, "Joint Source-Channel Coding for Asymmetric Slepian-Wolf Multiple Access Relay Channel," IEEE Wireless Communications Letters, vol. 6, no. 5, pp. 642-645, 2017.

[11] C. Hausl, F. Schreckenbach, I. Oikonomidis, and G. Bauch, "Iterative network and channel decoding on a tanner graph," 43rd Annual Allerton Conference on Communication, Control, and Computing, Monticello, USA, 2005.

[12] P. S. Lu, X. Zhou, K. Anwar, and T. Matsumoto, "Joint adaptive network-channel coding for energy-efficient multiple-access relaying," IEEE Transactions on Vehicular Technology, vol. 63, no. 5, pp. 22982305, 2014.

[13] R. Youssef, and A. G. i Amat, "Distributed serially concatenated codes for multi-source cooperative relay networks," IEEE Trans. Wireless Commun., vol. 10, no. 1, pp. 253-263, Jan. 2011.

[14] S. Ben Slimane, "A joint channel-network coding based on product codes for the multiple-access relay channel," ISRN Communications and Networking, 2012.

[15] J. He, V. Tervo, S. Qian, Q. Xue, M. Juntti, and T. Matsumoto, "Performance Analysis of Lossy Decode-and-Forward for Non-Orthogonal MARCs," IEEE Transactions on Wireless Communications, vol. 17, no. 3, pp. 1545-1558, 2018.

[16] X. Zhou, P. S. Lu, K. Anwar, and T. Matsumoto, "Correlated sources transmission in orthogonal multiple access relay channel: Theoretical analysis and performance evaluation," EEE Transactions on Wireless Communications, vol. 13, no. 3, pp. 1424-1435, 2014.

[17] F. P. Luus, and B. T. Maharaj, "Joint source-channel-network coding for bidirectional wireless relays," IEEE International Conference on Acoustics, Speech and Signal Processing (ICASSP), pp. 3156-3159, 2011.

[18] M. Fresia, F. Prez-Cruz, H. V. Poor, and S. Verdu, "Joint source and channel coding," IEEE Sig. Proc. Mag., vol. 27, no. 6, Nov. 2010.

[19] C. Chen, L. Wang, and S. Liu, “The Design of Protograph LDPC Codes as Source Codes in a JSCC System," IEEE Communications Letters, vol. 22, no. 4, pp. 672-675, 2018.

[20] M. Ben Abdessalem, A. Zribi, T. Matsumoto, and A. Bouallegue, "Graph-based Joint Source Channel LDPC decoding for cooperative communication with error-corrupted relay observations," Wireless Communications and Mobile Computing Conference (IWCMC), 13th International. IEEE, pp. 1588-1593, Valencia, Spain, Jun. 2017.

[21] R. Youssef, and A. G. i Amat, "Distributed serially concatenated codes for multi-source cooperative relay networks," IEEE transactions on wireless communications, vol. 10, no. 1, pp. 253-263, 2011. 